Modern Physics Letters A

(C) World Scientific Publishing Company

\title{
Time Variation of Fine Structure Constant and Proton-Electron Mass Ratio with Quintessence
}

\author{
Seokcheon Lee \\ Institute of Physics, Academia Sinica, \\ Taipei, Taiwan, 11529, R.O.C. \\ skylee@phys.sinica.edu.tw
}

Received (31 January 2007)

Revised (Day Month Year)

\begin{abstract}
Recent astrophysical observations of quasar absorption systems indicate that the fine structure constant $\alpha$ and the proton-electron mass ratio $\mu$ may have evolved through the history of the universe. Motivated by these observations, we consider the cosmological evolution of a quintessence-like scalar field $\phi$ coupled to gauge fields and matter which leads to effective modifications of the coupling constants and particle masses over time. We show that a class of models where the scalar field potential $V(\phi)$ and the couplings to matter $B(\phi)$ admit common extremum in $\phi$ naturally explains constraints on variations of both the fine structure constant and the proton-electron mass ratio.

Keywords: Time Varying Fine Structure Constant; Proton-Electron Mass Ratio; Quintessence.
\end{abstract}

PACS Nos.: include PACS Nos.

\section{Introduction}

Recent observations show non-vanishing time variation of the fine structure constant $\alpha$ by use of the relativistic shifts of atomic energy levels in quasar absorption spectra 1 and nontrivial time evolution of the proton-electron mass ratio $\mu=m_{p} / m_{e}$ from the observations of $\mathrm{H}_{2}$ spectral lines in quasars 2 .

However there have been attempts to detect a variation in $\alpha$ using similar methods 3 that has shown null results so far. This rather controversial situation becomes even more complicated if other constraints on $\Delta \alpha / \alpha$ are taken into account 4 . The Oklo natural reactor and meteoritic abundances of rhenium provide stringent constraints on the change of the coupling constants that goes back to $z \sim 0.1-0.4$ 51617. However, a recent re-analysis of Oklo phenomenon suggested that the present data are consistent with the non-zero change of $\Delta \alpha / \alpha=4.5 \times 10^{-8} \underline{8}$.

In spite of its questionable status of the non-zero claim for $\Delta \alpha / \alpha$ there have been a number of attempts $9 / 10|11 / 12| 13 / 14 \mid 15] 16|17| 18$ to build simple models that could account for a possible $O\left(10^{-5}\right)$ relative shift in the fine structure constant at redshifts $z \sim 1$. The Bekenstein model 13 with a scalar field coupled to both the 


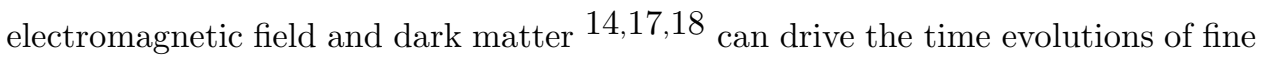
structure constant and masses.

In quintessence models, which is one of the most commonly proposed candidates for dark energy $19 \mid 20$ to explain the current accelerating universe 21 we can naturally have the change of the coupling constant $22|23| 24|25| 26|27| 28|29| 30$ and masses of particles $31 \mid 32$ over cosmological times due to the interaction of quintessence field with gauge fields and matter.

In this paper we consider the scalar field in the Bekenstein model as a quintessence field to check the time variations of the fine structure constant and

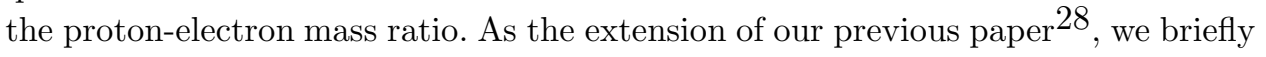
show the result of the cosmological evolution of the dark energy and dark matter energy density over the redshift. We show that the cosmology of coupled quintessence models with a common extremum in $V(\varphi)$ and gauge and matter/gauge couplings $B_{i}(\varphi)$ which can be consistent with all observational requirements. This paper is organized as follows. In the next section we introduce our model and display the necessary cosmological equations. In sections 3 , we make predictions for the variation of the coupling constants. We consider the time varying masses in section4. Section 5 is devoted to the conclusions.

\section{Cosmological Evolution of the Coupled Scalar Field}

The action including the interaction of a dimensionless light scalar field $\phi$ with matter and gauge fields is given by,

$$
\begin{aligned}
S_{\phi}= & \int d^{4} x \sqrt{-g}\left\{\frac{\bar{M}^{2}}{2}\left[\partial^{\mu} \phi \partial_{\mu} \phi-R\right]-V(\phi)-\frac{B_{F i}(\phi)}{4} F_{\mu \nu}^{(i)} F^{(i) \mu \nu}\right. \\
& \left.+\sum_{j}\left[\bar{\psi}_{j} i \not D \psi_{j}-B_{j}(\phi) m_{j} \bar{\psi}_{j} \psi_{j}\right]\right\}
\end{aligned}
$$

where $\bar{M}=M_{p} / \sqrt{8 \pi}$ is the reduced Planck mass, $B_{F i}(\phi)$ represents the $\phi$ dependence of the gauge couplings in Standard Model where the sum is over all three groups. $\psi_{j}$ represents both Standard Model fermions and other matter field (i.e. scalar Higgses, Majorana neutrinos etc.). Since $\phi$ couples to the trace $T_{\mu}^{\mu}$ of dark matter, our results are independent of the nature of the dark matter particles (scalars or fermions).

Given a potential $V(\phi)$, the perfect fluid energy density and pressure contributions due to $\phi$ are:

$$
\begin{aligned}
\rho_{\phi} & =\frac{1}{2} \bar{M}^{2} \dot{\phi}^{2}+V(\phi) \\
p_{\phi} & =\frac{1}{2} \bar{M}^{2} \dot{\phi}^{2}-V(\phi) \equiv \omega_{\phi} \rho_{\phi},
\end{aligned}
$$

where the parameter $\omega_{\phi}$ is the equation of state (EOS) of the scalar field. By including the scalar field, we have the following Friedmann equation in a Robertson-Walker 
Universe,

$$
H^{2}=\frac{1}{3 \bar{M}^{2}}\left(\rho_{\phi}+\rho_{r}+\rho_{m}\right) \equiv \frac{1}{3 \bar{M}^{2}} \rho_{c r},
$$

where $H=\dot{a} / a$ is the Hubble expansion rate, $\rho_{r}$ and $\rho_{m}$ is the energy density of radiation and matter, and $\rho_{c r}$ is the critical energy density. Notice the $\phi$-dependence of the energy density of matter, i.e. $\rho_{m}=\sum_{j} B_{j}(\phi) m_{j}<\bar{\psi}_{j} \psi_{j}>\equiv \sum_{j} B_{j}(\phi) m_{j} n_{j}$. Using these definitions, we can write the scalar field equation,

$$
\ddot{\phi}+3 H \dot{\phi}+\frac{1}{\bar{M}^{2}} \frac{\partial V}{\partial \phi}=-\frac{1}{\bar{M}^{2}} \frac{\partial \ln B_{m}}{\partial \phi} \rho_{m} .
$$

For cosmological studies that span a large range of redshifts $z$, it is convenient to introduce the variable $x$ as the logarithm of the scale factor $a$,

$$
x=\ln a=-\ln (1+z)
$$

where we choose the present scale factor $a^{(0)}=1$. With the use of the variable $x$, we can rewrite the relevant system of equations for $d \ln \rho_{i} / d x$ in the following form,

$$
\begin{aligned}
\frac{d \ln \rho_{m}}{d x} & =-3\left(1+\omega_{m}\right)+\frac{\partial \ln B_{m}(\phi)}{\partial \phi} \frac{d \phi}{d x}, \\
\frac{d \ln \rho_{r}}{d x} & =-3\left(1+\omega_{r}\right), \\
\frac{d \ln \rho_{\phi}}{d x} & =-3\left(1+\omega_{\phi}\right)-\frac{\partial \ln B_{m}(\phi)}{\partial \phi} \frac{d \phi}{d x} \frac{\rho_{m}}{\rho_{\phi}},
\end{aligned}
$$

where $\omega_{r}=1 / 3$ and $\omega_{m}=0$ should be used for radiation and matter respectively.

The derivative of $\phi$ with respect to $x$ can be obtained from Eq (2),

$$
\left(\frac{d \phi}{d x}\right)^{2}=3 \Omega_{\phi}\left(1+\omega_{\phi}\right)
$$

If $\omega_{\phi}$ is close to -1 , the kinetic energy of the scalar field goes to zero which occurs when the scalar field is close to the minimum of the potential.

When the change in $B_{m}(\phi)$ is not small and cannot be neglected, the scaling of the matter energy density differs from usual $a^{-3}$ behavior because of the changing mass, due to $B_{m}(\phi)$, while the scaling of radiation energy density remains unchanged,

$$
\begin{aligned}
\rho_{m}(x) & =\rho_{m}^{(0)} a^{-3} \frac{B_{m}(\phi(x))}{B_{m}(\phi(0))}, \quad \text { and } \quad \rho_{r}(x)=\rho_{r}^{(0)} a^{-4} \\
\rho_{r} & =\frac{a_{e q}}{a} \frac{B_{m}\left(\phi\left(x_{e q}\right)\right)}{B_{m}(\phi(x))} \rho_{m}
\end{aligned}
$$


4 Seokcheon Lee
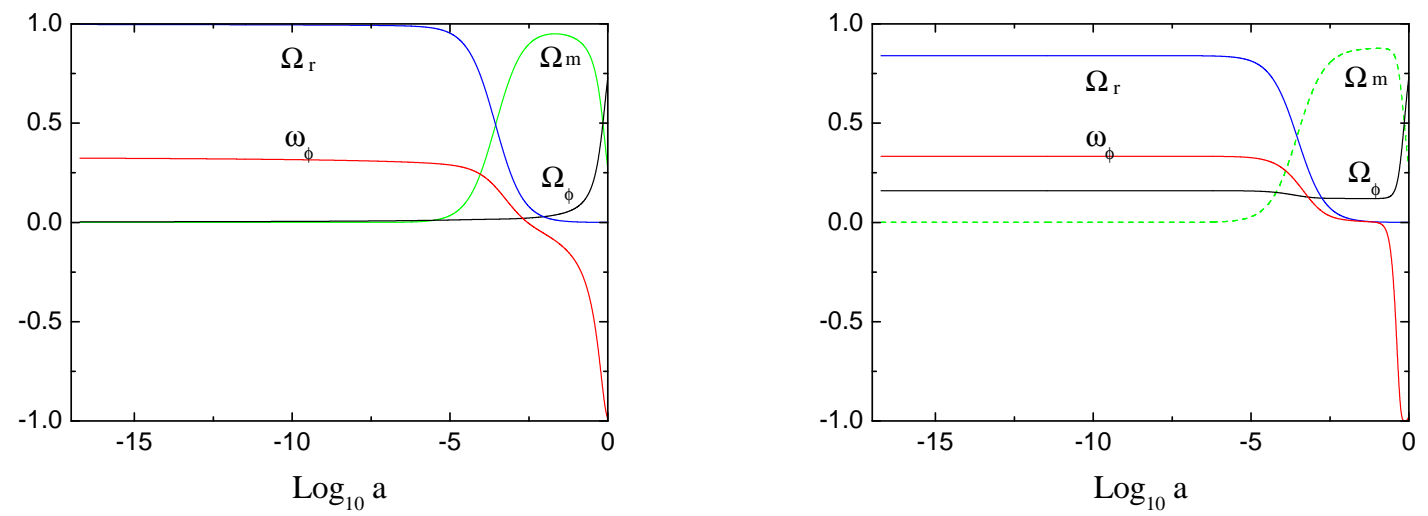

Fig. 1. The cosmological evolution of the equation of state parameter, $\omega_{\phi}$, and the energy density parameters, $\Omega_{i}$, of each component for $\lambda=5$ when $n=10^{-3}$. a) When the potential is $V=$ $V_{0} \exp \left(\lambda \phi^{2} / 2\right)$. b) For the $V=V_{0} \cosh (\lambda \phi)$ potential.

With the use of these relations, we find

$$
\begin{aligned}
& \frac{d \ln \left(1-\Omega_{\phi}\right)}{d x}=\Omega_{\phi}\left[3 \omega_{\phi}-\left(\frac{a_{e q}^{(c)}}{a+a_{e q}^{(c)}}\right)\right]+\left(\frac{a}{a+a_{e q}^{(c)}}\right) \frac{\partial \ln B_{m}}{\partial \phi} \frac{d \phi}{d x} \\
& \frac{d \ln \left(1-\omega_{\phi}\right)}{d x}=3\left(1+\omega_{\phi}\right)+\frac{\partial \ln V}{\partial \phi} \frac{d \phi}{d x}+\frac{\left(1-\Omega_{\phi}\right)}{\Omega_{\phi}}\left(\frac{a}{a+a_{e q}^{(c)}}\right) \frac{\partial \ln B_{m}}{\partial \phi} \frac{d \phi}{d x}
\end{aligned}
$$

where we have introduced an auxiliary function, $a_{e q}^{(c)}(\phi)=a_{e q} B_{m}\left(\phi\left(x_{e q}\right)\right) B_{m}^{-1}(\phi(x))$.

We assume that all functions $B_{i}(\phi)$ and $V(\phi)$ admit a common extremum, which is a generalization of Damour-Nordtvedt and Damour-Polyakov constructions 33 [34. Near this extremum, all functions admit an expansion

$$
B_{i}(\phi)=1+\zeta_{i} \phi+\frac{1}{2} \xi_{i} \phi^{2}+\ldots ; \quad V(\phi)=V_{0}\left(1+\lambda \phi+\frac{1}{2} \lambda \phi^{2}+\ldots\right),
$$

where $\zeta, \xi_{i}$ and $\lambda$ are dimensionless parameters, while $V_{0}$ is of the order of the dark energy density today.

We consider two simple potentials $V(\phi)$ and follow the same ansatz for functions $B_{i}(\phi)$ as in our previous work 28 .

$$
\begin{aligned}
\text { case A : } \quad V(\phi) & =V_{0} \exp \left(\frac{\lambda \phi^{2}}{2}\right) \\
\text { case B : } \quad V(\phi) & =V_{0} \cosh (\lambda \phi) \\
B_{i}(\phi)=\left(\frac{b_{i}+V(\phi) / V_{0}}{1+b_{i}}\right)^{n_{i}}, & \text { where } b_{i}+1>0
\end{aligned}
$$

We show the cosmological evolution of the equation of state parameter, $\omega_{\phi}$, and the energy density parameters, $\Omega_{i}$, of each component for both potentials in Fig. 1. 

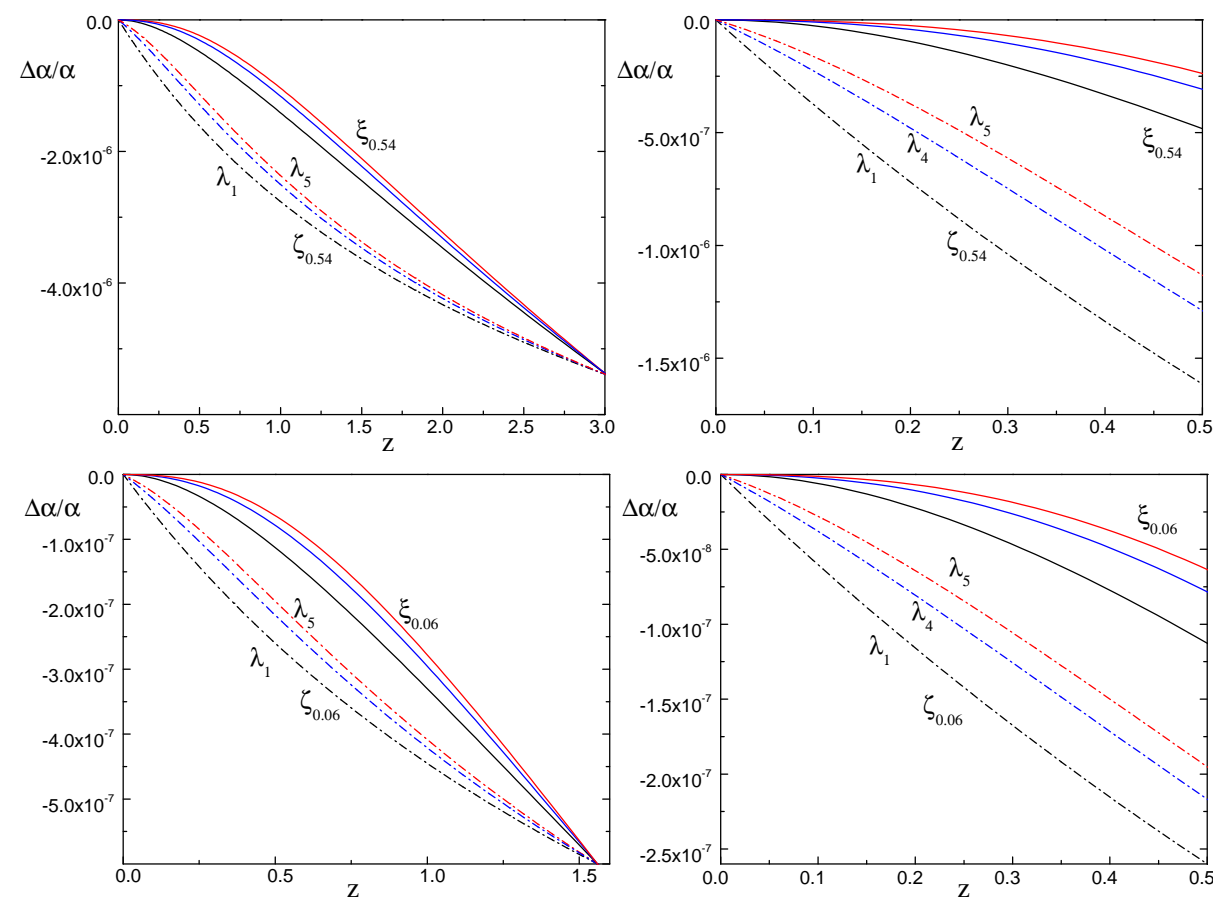

Fig. 2. a. The evolution of $\Delta \alpha / \alpha$ over the redshift range $0 \leq z \leq 3$ driven by the potential (16). Panels a) and b) use the common normalization $\Delta \alpha / \alpha=-0.54 \times 10^{-5}$ at $z=3$. Figures c) and d) use the common normalization $\Delta \alpha / \alpha=-0.06 \times 10^{-5}$ at $z=1.5$. The solid lines correspond to the choice $\zeta=0$ and $\xi \neq 0$, whereas the dashed-dotted lines allow $\zeta \neq 0$.

In this figure we use $\lambda=5$ and $n=10^{-3}$ which is a reasonable value adopted from previous work 35 .

\section{Time Variation of the Fine Structure Constant}

To study the cosmological evolution of the fine structure constant we use the following relation between $B_{F}$ and $\alpha$,

$$
\frac{\Delta \alpha(z)}{\alpha} \equiv \frac{\alpha(z)-\alpha(0)}{\alpha(0)}=\frac{B_{F}(\phi(0))}{B_{F}(\phi(z))}-1 .
$$

Note that the present value of the field $\phi(0)$ is close to zero.

In Fig. 2 and Fig. 3, we show the late-time evolution of $\alpha$ for the potentials considered in the previous section. These figures are adopted from the previous works 28 . For the late time evolution of $\alpha$ we can use the expansion of $B_{F}(\phi)$ given in (15). In this case, the expression for $\Delta(\alpha)$ takes the following simple form,

$$
\frac{\Delta \alpha(z)}{\alpha}=\frac{\xi}{2}\left(\phi^{2}(0)-\phi^{2}(z)\right),
$$



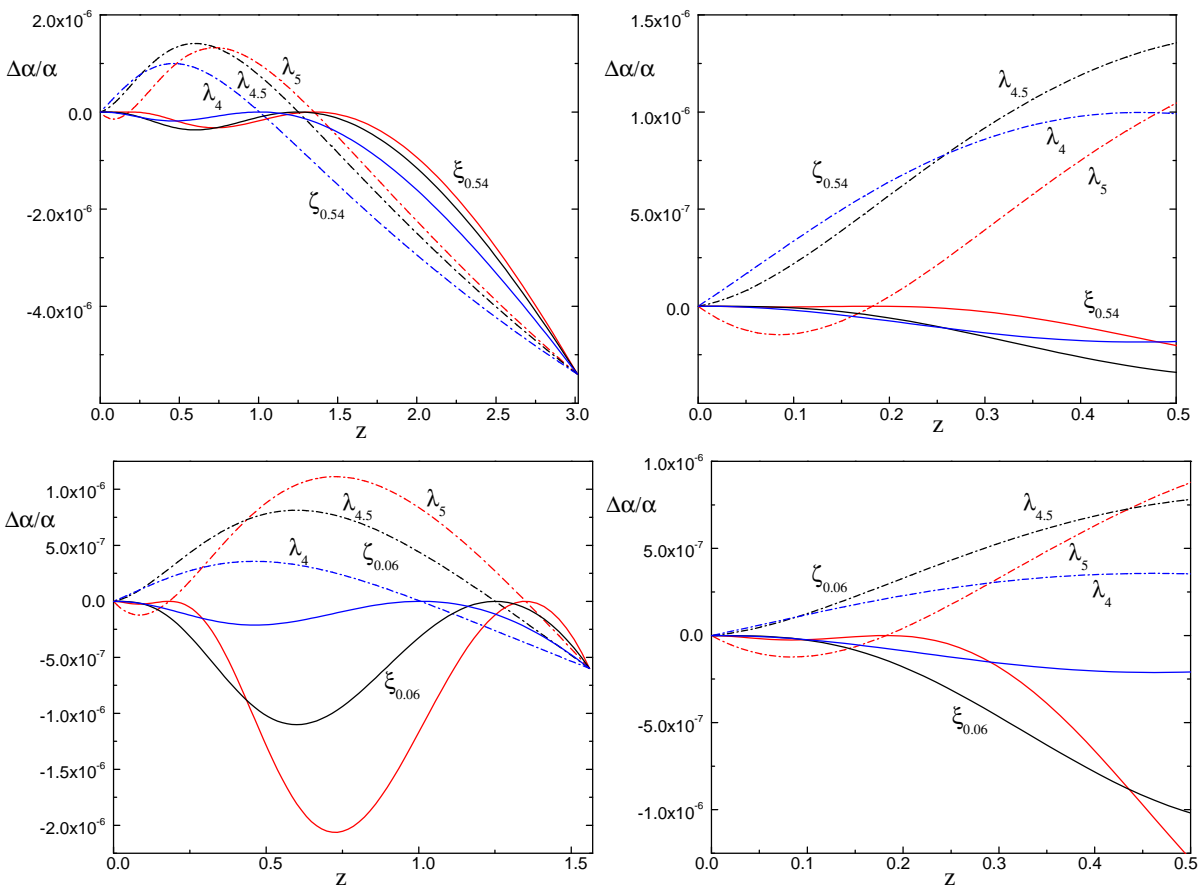

Fig. 3. As in Fig. 2 for the potential 17 Here $\lambda=4,4.5$, and 5 .

where we dropped the subscript $F$ in $\xi_{F}$ to be concise. Using the result of the previous section, we can predict the evolution of $\alpha$ over redshift in terms of two parameters, $\xi$ and $\lambda$. We choose two characteristic values of $\xi$, based on two QSO results. To be consistent with the non-zero result for $\Delta \alpha$ by Murphy et al. 1], we choose $\xi$ in such a way that $\Delta \alpha / \alpha=-5.4 \times 10^{-6}$ at a redshift of 3 . Another option that we explore is $|\Delta \alpha / \alpha| \leq 6 \times 10^{-7}$ at $z=1.5$, which is motivated by the

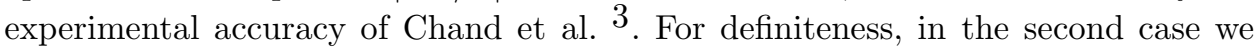
choose $\Delta \alpha / \alpha=-7 \times 10^{-6}$.

\section{Time Variation of The Proton-Electron Mass Ratio}

The proton-electron mass ratio evolves by the following simple relation between $B_{m}$ and $\mu$ in our consideration,

$$
\frac{\Delta \mu}{\mu} \equiv \frac{m_{p}(z) / m_{e}(z)-m_{p}(0) / m_{e}(0)}{m_{p}(0) / m_{e}(0)}=\frac{B_{p}(\phi(z))}{B_{e}(\phi(z))} \frac{B_{e}(\phi(0))}{B_{p}(\phi(0))}-1 .
$$

We can find the coupling strength $n$ for the each potential when we adopt the result of one of the recent observation 3 around $z \sim 2.8$,

$$
\frac{\Delta \mu}{\mu}=(2.4 \pm 0.6) \times 10^{-5} \text {. }
$$


Time Varying Fine Structure Constant and Proton-Electron Mass Ratio with Quintessence 7
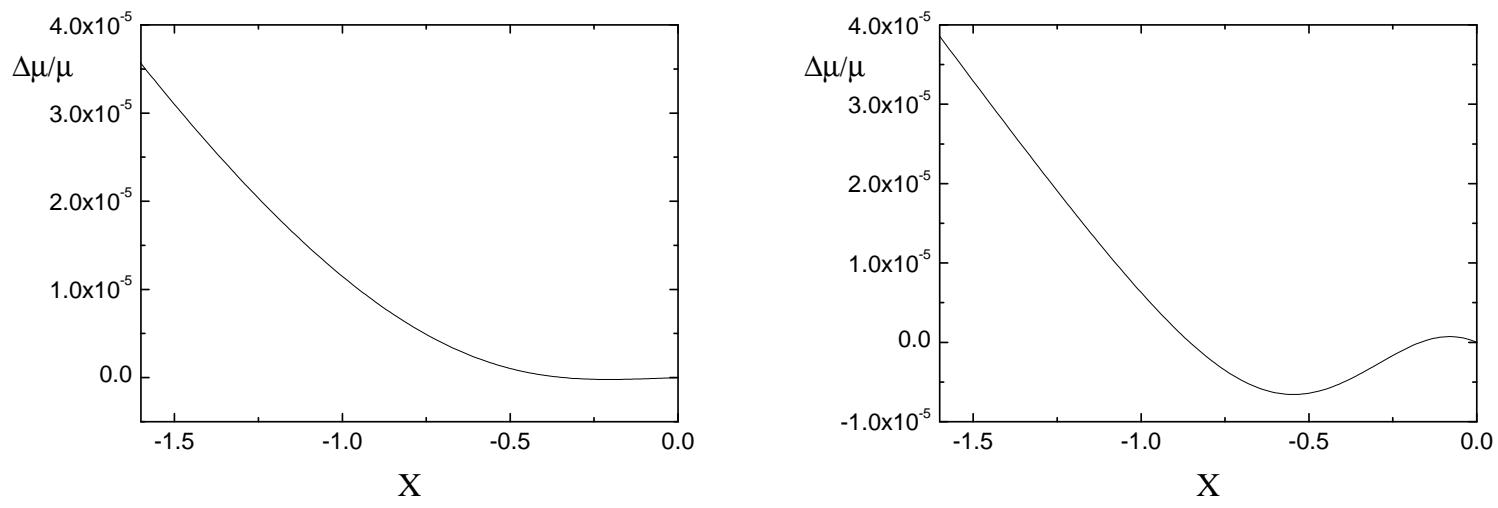

Fig. 4. The cosmological evolution of the proton-electron mass ratio $\Delta \mu / \mu$. a) For the $\exp \left(\lambda \phi^{2} / 2\right)$ potential, $n=2.5 \times 10^{-5}$. b) $n=-2.8 \times 10^{-5}$ for the $\cosh (\lambda \phi)$ potential.

In Fig. 4, we show the cosmological evolution of the proton-electron mass ratio with the derived coupling constants $n$ for each potential. In the left panel of the figure we show the cosmological evolution of $\Delta \mu / \mu$ for the exponential potential. We find the coupling constant $n=2.5^{-5}$ for this potential. The right panel of the figure shows the time variation of $\Delta \mu / \mu$ for the cosh-potential where we find $n=-2.8 \times 10^{-5}$.

\section{Conclusions}

We have analyzed the cosmological evolution of the scalar field driven by its selfinteraction potential, $V(\phi)$, and its possible couplings to matter, $B_{m}(\phi)$.

We have seen that the coupling of the scalar field to the electromagnetic field and to matters can explain the recent observations. They can explain the time evolutions of the fine structure constant and the proton-electron mass ratio. Our models by use of quintessence couplings to gauge fields and matters can pass the all the current known experimental limits.

\section{Acknowledgments}

We thank CosPA2006 organizing committee for their hospitality and for organizing such a nice meeting.

\section{References}

1. J. K. Webb et al., Phys. Rev. Lett. 87, 091301 (2001); M. T. Murphy, J. K. Webb and V. V. Flambaum, Mon. Not. Roy. Astron. Soc. 345, 609 (2003); P. Tzanavaris, M. T. Murphy, J. K. Webb, V. V. Flambaum and S. J. Curran, Mon. Not. Roy. Astron. Soc. 374, 634 (2007); M. T. Murphy, J. K. Webb and V. V. Flambaum arXiv: astroph/0612407 (2006). 
2. W. Ubachs and E. Reinhold, Phys. Rev. Lett. 92, 101302 (2004); P. Tzanavaris, J. K. Webb, M. T. Murphy, V. V. Flambaum and S. J. Curran, Phys. Rev. Lett. 95, 041301 (2005); E. Reinhold et al., Phys. Rev. Lett. 96, 151101 (2006).

3. H. Chand, R. Srianand, P. Petitjean and B. Aracil, Astron. Astrophys. 417, 853 (2004); R. Srianand, H. Chand, P. Petitjean and B. Aracil, Phys. Rev. Lett. 92, 121302 (2004).

4. For a review see: J. P. Uzan, Rev. Mod. Phys. 75, 403 (2003).

5. A. I. Shlyakhter, Nature 264, 340 (1976); T. Damour and F. Dyson, Nucl. Phys. B 480, 37 (1996); Y. Fujii et al., Nucl. Phys. B 573, 377 (2000).

6. K. A. Olive, M. Pospelov, Y. Z. Qian, A. Coc, M. Casse and E. Vangioni-Flam, Phys. Rev. D 66, 045022 (2002); K. A. Olive, M. Pospelov, Y. Z. Qian, G. Manhes, E. Vangioni-Flam, A. Coc and M. Casse, Phys. Rev. D 69, 027701 (2004).

7. Y. Fujii and A. Iwamoto, Phys. Rev. Lett. 91, 261101 (2003).

8. S. K. Lamoreaux, Phys. Rev. D 69, 121701 (2004).

9. T. Chiba and K. Kohri, Prog. Theor. Phys. 107, 631 (2002).

10. C. Wetterich, Phys. Lett. B 561, 10 (2003) ; arXiv : hep-ph/0302116.

11. L. Anchordoqui and H. Goldberg, Phys. Rev. D 68, 083513 (2003).

12. E. J. Copeland, N. J. Nunes and M. Pospelov, Phys. Rev. D 69, 023501 (2004).

13. J. D. Bekenstein, Phys. Rev. D 25, 1527 (1982).

14. T. Damour, G. W. Gibbons and C. Gundlach, Phys. Rev. Lett. 64, 123 (1990).

15. M. Livio and M. Stiavelli, Ap. J. Lett. 507, L13 (1998).

16. S. J. Landau and H. Vucetich, S. J. Landau and H. Vucetich, Astrophys. J. 570, 463 (2002).

17. K. A. Olive and M. Pospelov, Phys. Rev. D 65, 085044 (2002).

18. H. B. Sandvik, J. D. Barrow and J. Magueijo, Phys. Rev. Lett. 88, 031302 (2002); J. D. Barrow, H. B. Sandvik and J. Magueijo, Phys. Rev. D 65, 063504 (2002); J. D. Barrow, J. Magueijo and H. B. Sandvik, Phys. Lett. B 541, 201 (2002).

19. B. Ratra, P.J.E. Peebles, Phys. Rev. D 37, 3406 (1988); Ap. J. Lett 325, 117 (1988); C. Wetterich, Nucl. Phys. B 302, 668 (1988); R.R. Caldwell, R. Dave and P.J. Steinhardt, Phys. Rev. Lett 80, 1582 (1998).

20. P.G. Ferreira and M. Joyce, Phys. Rev. D 58, 023503 (1998).

21. A. G. Riess et al. [Supernova Search Team Collaboration], Astron. J. 116, 1009 (1998); S. Perlmutter et al. [Supernova Cosmology Project Collaboration], Astrophys. J. 517, 565 (1999); N. A. Bahcall, J. P. Ostriker, S. Perlmutter and P. J. Steinhardt, Science 284, 1481 (1999).

22. T. Chiba and K. Kohri Prog. Theor. Phys. 107, 631 (2002).

23. J. D. Barrow, J. Magueijo and H. B. Sandvik Phys.Lett. B 541, 201 (2002).

24. C. Wetterich, JCAP 0310, 002 (2003); Phys. Lett. B 561, 10 (2003).

25. D. S. Lee, W. Lee and K. W. Ng, Int. J. Mod. Phys. D 14, 335 (2005).

26. N. J. Nunes and J. E. Lidsey, Phys. Rev. D 69, 123511 (2004).

27. P. P. Avelino, C. J. A. Martins and J. C. R. Oliveira, Int. J. Mod. Phys. D bf 14, 677 (2005).

28. S. Lee, K. A. Olive and M. Pospelov, Phys. Rev. D 70, 083503 (2004).

29. V. Marra and F. Rosati, JCAP 0505, 011 (2005).

30. P. P. Avelino, C. J. A. P. Martins, N. J. Nunes and K. A. Olive, Phys.Rev. D 74, 083508 (2006).

31. J. D. Barrow, Phys. Rev. D 71, 083520 (2005); Phys. Rev. D 72, 043521 (2005).

32. T. Chiba, T. Kobayashi, M. Yamaguchi and J. Yokoyama arXiv:hep-ph/0610027 (2006).

33. T. Damour and K. Nordtvedt, Phys. Rev. Lett. 70, 2217 (1993); Phys. Rev. D 48, 3436 (1993). 
34. T. Damour and A.M. Polyakov, Nucl. Phys. B 423, 532 (1994) Gen. Rel. Grav 26, 1171 (1994).

35. S. Lee, G.-C. Liu, K-W. Ng, Phys. Rev. D 73, 083516 (2006).

36. R. Marnelius, Acta Phys. Pol. B 13, 669 (1982).

37. J. D. Bjorken, in Lecture Notes on Current-Induced Reactions, eds. J. Komer et al. (Springer, 1975).

38. A. Bohr and B. R. Mottelson, Nuclear Structure (Benjamin, 1969), Vol. 1, pp. 100-102.

39. T. Toimela, Helsinki Research Institute for Theoretical Physics, Report No. HU-TFT82-37, 1982 (unpublished). 